\author{
Military Technical College \\ Kobry El-Kobbah, \\ Cairo, Egypt
}

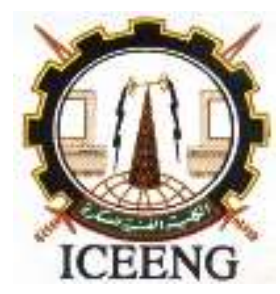

\author{
$11^{\text {th }}$ International Conference \\ on Electrical Engineering \\ ICEENG 2018
}

\title{
Roads Feature Extraction from High Resolution Satellite Images Using Decision Tree Classifier
}

\author{
Mahmoud abdallah Shwaky *, Fawzy Eltohamy Hassan Amer *, and Ahmed S. \\ Elsharkawy *
}

\begin{abstract}
Extracting roads network data, from satellite images, is important for urban planning, infrastructure development, navigation applications, military purposes and updating topographic GIS databases. Very high spatial resolution remote sensing images are useful source for extracting roads information. In this paper a novel algorithm for roads feature extraction of sub-urban and rural areas from high resolution images is developed. The developed algorithm is based on Decision Tree classifier (DTC). The developed DTC models are trained and applied on corrected Multispectral images acquired by Worldview - II (WV-2) satellite. The experimental work is performed using MATLAB with Graphic User Interface (GUI) for designing and managing the training data. The result of DTC model were evaluated using three of quality measures. The results are compared with a digitized reference roads layer. The obtained results show the possibility of using the developed algorithm for automatic roads feature extraction from high resolution images. The results provide about $82 \%$ accuracy of roads extraction.
\end{abstract}

KEY WORDS

Indices, Reflectance, Roads extraction, Assessment.

\section{Introduction}

The roads extraction from RS images is a challenging but important research topic. Roads are essential objects of transportation; and give many different supports for human civilization. The research of road extraction is of great significance for traffic management, city planning, road monitoring, GPS navigation and map updating [1]. Different techniques such as Knowledge-based methods, Mathematical morphology methods, Active contour model, Classification-based methods are used to extract

\footnotetext{
* Egyptian Armed Forces.
} 
roads from satellite images [2]. Satellite remote sensing images provides qualitative and quantitative information which reduces time for development and complexity of field work [3]. In addition remote sensing Satellites collect data/images at regular intervals. Therefore the amount of received data is huge and it is growing exponentially as the technology is growing at rapid speed as timely and data volumes have been growing at exponential rate [4]. In practice, the RS image quality can be affected by different factors such as the sensor type, spectral and spatial resolution, weather conditions, light variation, and ground characteristic so interference factors must be considered [5]. Using corrected high resolution sensor such as that of WV-2 allows greater automated classification with reduced manual editing. Different road features in an image have different properties for road extraction. Geometric features have the direct relationships with the road shapes. Photometric features are close to the road gray levels or colors. Topological features and functional features [6] are relatively simple but hard to apply in real applications.. Satellite image classification plays a vital role in interpretation and extract of valuable information from volumes satellite images. DTC algorithm with fixed thresholds against multi-temporal high resolution satellite imagery is one of the most important techniques for monitoring place at different times the training data from one time and place are applied to classifying remotely sensed data in other place and time. It is needed obtaining available information of sensor and good study for spectral profile of classes in different bands to provide automated and robust algorithm [7]. 


\section{Concept of the proposed roads extraction algorithm}

A feature extraction from an image simply means the search of the presence of this feature. Generally mathematical operations that combine the pixels digital values (DN) of two or more bands (layers) in MS images can be measures of the presence of specific feature. For example, the calculation of: (DN) IR - (DN) R gives a simple, yet very useful measure of the presence of vegetation [8]. Such mathematical combinations of the DN values of different bands create output images. More complex combinations can be for examples:

$$
\frac{\text { Band } X-\text { Band } Y}{\text { Band } X+\text { Band } Y} \quad \frac{\text { Band } X}{\text { Band } Y}
$$

These indices are ratios of $X$ and $Y$ bands $D N$ values. These ratio images are derived from the absorption/reflection spectra of the material of interest. The absorption/reflection is based on the molecular bonds in the material surface. Thus, the ratio often gives information on the chemical composition of the feature. In this paper, the feature extraction process is performed using new proposed bands ratios. The proposed ratios are based on careful study of spectral profiles of the five classes (features), as given in details in section 5.2, present in the study area (water, vegetation, roads, shadows and buildings). These ratios are:

$$
(\mathrm{R}-\mathrm{NIR} 1) /(\mathrm{R}+\mathrm{NIR} 1) \quad \text { (For water and vegetation) }
$$

$$
\text { (CB - NIR2)/(CB - NIR2) (For shadow, roads and building) }
$$

Where R, NIR1, CB and NIR2 are operating spectral bands of the satellite WV-2

\section{Study area}

The study area is a subset of Rio de Janeiro state in the southeast of Brazil, fig (1). It is approximate $4 \mathrm{~km}^{2}$. It is bounded on the east and south by the South Atlantic Ocean .Rio de Janeiro has an area of $43,653 \mathrm{~km}^{2}$. The ground features in the selected area are: water, vegetation, roads, shadows and buildings.

\section{Remote sensing data of study area}

Data from WV-2 satellite is selected because it provides four new spectral bands: Coastal blue, Yellow, Red edge and NIR-2. These bands give additional information for different features which can lead to better feature extraction results [9]. The 
available data of the study area are three WV-2 satellite multispectral images (8 bands), Fig.2, Fig.3 and Fig.4. The images are acquired on January 19th, 2010. The projection of each image is UTM, with datum of WGS84 zone $23 \mathrm{~N}$. The upper left corner and lower right corner coordinates of each image are (lat $22^{\circ} 54 \backslash 7.79 \| \mathrm{S}$, lon $43^{\circ} 10 \backslash 38.09 \backslash \backslash \mathrm{W}$ ) and (lat $22^{\circ} 55 \backslash 11 \backslash \mathrm{S}$, lon $43^{\circ} 9 \backslash 28.2471 \backslash \backslash \mathrm{W}$ ) respectively. The images are acquired at the conditions givens in Table (1).

Table 1. Meta data of input images

\begin{tabular}{l||l||l||l}
\hline \hline \multicolumn{1}{l||}{ Specifications } & Image I & Image II & Image III \\
\hline \hline time of imaging & $13: 09: 53.5$ & $13: 10: 43.76$ & $13: 11: 58.76$ \\
\hline \hline $\begin{array}{l}\text { Mean sun elevation } \\
\text { angle }\end{array}$ & $63.5^{\circ}$ & $63.7^{\circ}$ & $64^{\circ}$ \\
\hline \hline Mean of nadir & $29.8^{\circ}$ & $7.5^{\circ}$ & $26.7^{\circ}$ \\
\hline \hline Mean track angle & $29.8^{\circ}$ & $7.6^{\circ}$ & $26.7^{\circ}$ \\
\hline \hline Mean cross angle & $1.6^{\circ}$ & $0.0^{\circ}$ & $-2.3^{\circ}$ \\
\hline \hline
\end{tabular}

\section{Experiment steps}

The purpose of this experiment is road features extractions for a given study area. The experiment steps are given in Fig. (5).

\subsection{Conversion of image pixels digital numbers (DN) to reflectance values}

Before feature extraction a conversion process of pixel digital numbers (DN) to reflectance values is performed to obtain accurate quantitative information from multispectral data of WV-2 satellite. It is executed using MATLAB R2015A, [11]. The conversion from radiometrically corrected image pixels to spectral radiance is done using equation (3), [10],for each band of the image data.

Where

$\boldsymbol{L}_{\lambda_{\text {pixel,band }}}$

$\boldsymbol{K}_{\text {band }}$

$\boldsymbol{q}_{\text {pixel,band }}$

$\Delta \lambda_{\text {band }}$

$$
L_{\lambda_{\text {pixel,band }}}=\frac{k_{\text {band }} \cdot q_{\text {pixel,band }}}{\Delta \lambda_{\text {band }}}
$$

\subsection{Construction of the spectral profile}

To construct the spectral profiles of the different classes the values of random points in study area for the five classes, are taken. Then the reflectance spectrum of each point in each class for the 8 bands is plotted against the wavelength, from Fig. (6) to Fig. (10), using ENVI 4.5. 


\subsection{Applying the proposed new band ratio}

The proposed band ratios (1) and (2) are applied on the 4 bands (R, NIR1, CB and NIR2) of the satellite images of the study area. The output images are normalized images with pixel values from $(-1: 1)$.

\subsection{Classification of output normalized images}

R1 bands ratio is first applied to separate the image into vegetation and water within lower and upper thresholds, where water is detected above certain threshold. $\mathrm{R} 2$ bands ratio is then applied to detect shadow above certain threshold. Finally the roads and buildings are detected above certain threshold. Fig. 11 summarizes this step, indicating the calculated threshold values. The threshold values are calculated according to minimum and maximum value in the normalized image for every feature. The results of feature extraction are given in Fig. 12 to Fig. 14.

\subsection{Roads extraction}

Attribute selection is more commonly applied to vector analysis. DTC aggregates spatial information into groups or classes. The result of selection of class number 5 (roads) from classified images is given in Fig. 15

\section{Result and Assessment}

The automatically extracted roads are compared with manually digitized reference roads, Fig. 16 to perform accuracy assessment. Because roads are linear features, it is possible to use all the data rather than just sample points to conduct the accuracy assessment in a GIS data. There are several metrics to evaluate the quality of extracted roads. In this paper the following three measures for accuracy assessment of road extraction results are applied [11]:

$$
\begin{aligned}
& \text { Completeness }=\frac{\text { length of } \text { matched extraction }}{\text { length of reference }} \\
& \text { Correctness }=\frac{\text { length of } \text { matched extraction }}{\text { length of extraction }} \\
& \text { Quality }=\frac{\text { length of matched extraction }}{\text { length of extraction+length of unmatchedrefrence }}
\end{aligned}
$$

Completeness represents the percentage of reference data being correctly extracted. Correctness indicates the percentage of correctly extracted roads. Therefore, completeness is producer's accuracy and Correctness is users' accuracy. The quality represents the overall accuracy[12]. 
Table (2) Assessment results of the feature extraction techniques

\begin{tabular}{l||c||c}
\hline \hline & Extracted Road & Reference \\
\hline \hline Matched & 20155.274 & 21024.574 \\
\hline \hline Unmatched & 2701.135 & 1485.899 \\
\hline \hline Total length $(\mathrm{m})$ & 22856.409 & 22510.473 \\
\hline \hline Completeness & \multicolumn{2}{|c}{0.895} \\
\hline \hline Correctness & \multicolumn{2}{|c}{0.882} \\
\hline \hline Quality & \multicolumn{2}{|c|}{0.827} \\
\hline \hline
\end{tabular}

Assessment results of the road extraction from WV-2 images show that the completeness is $89 \%$ and correctness is $88 \%$. This means that $89 \%$ of the roads were successfully extracted and $88 \%$ of extracted road were correct. Shadows and bad contrast contribute to un-extracted roads and lead to low completeness. Building roofs or other objects with similar reflective values cause low correctness.

\section{Conclusion}

There are still some problems with the automatic extraction of overclouded roads, which is the main reason for errors in road extraction results. It is possible to use a DTC technique to separate the intersection of different objects from roads. Driveways are useful detailed information for some agencies the accuracy assessments for these three tested images show that the proposed algorithm gives good result for rural and suburban areas; the completeness is greater than $85 \%$. The proposed algorithm can give better accuracy when extracting roads from images with good contrast. It has great potential for updating GIS data and commercial value.

\section{Figures}

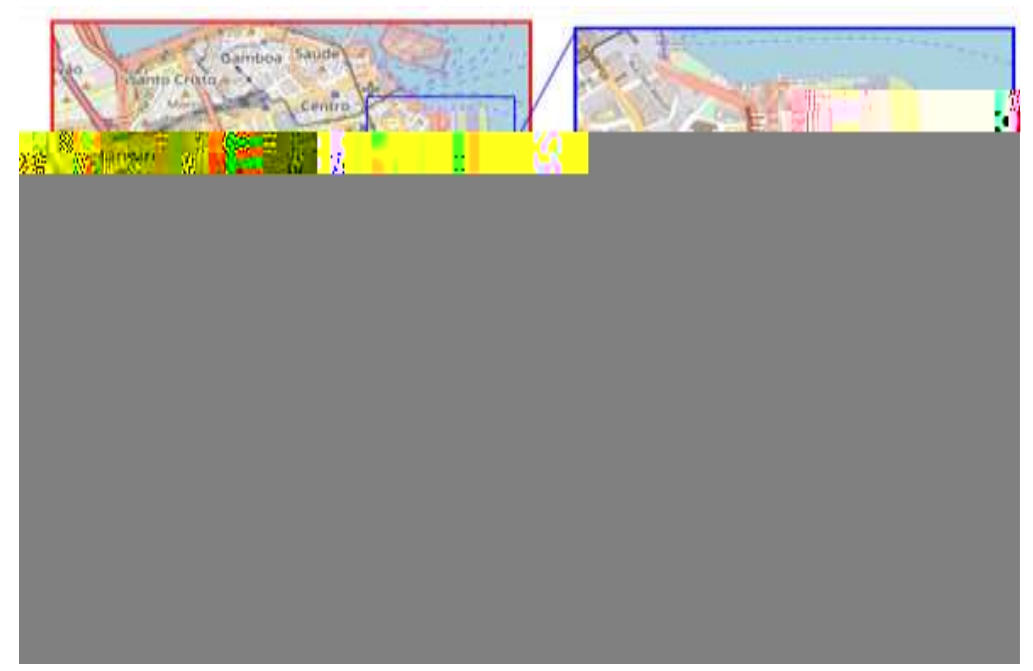

Fig. 1: Location of the selected area of Rio de Janeiro city in Brazil. 


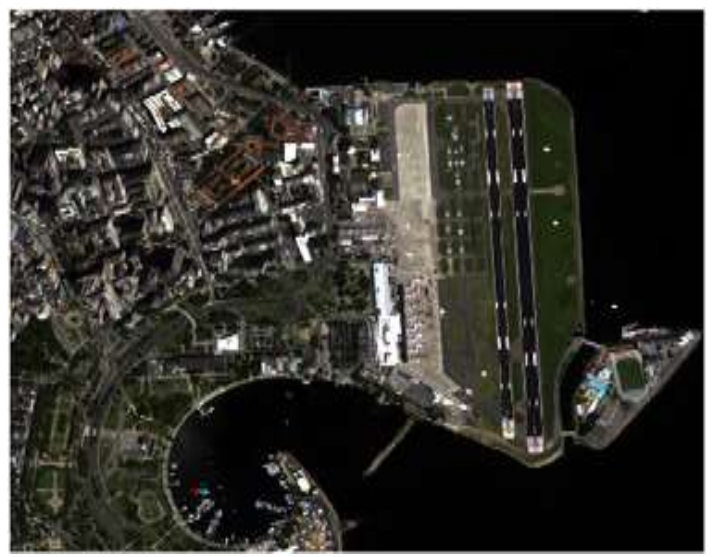

Fig. 2: Selected area, image (I).

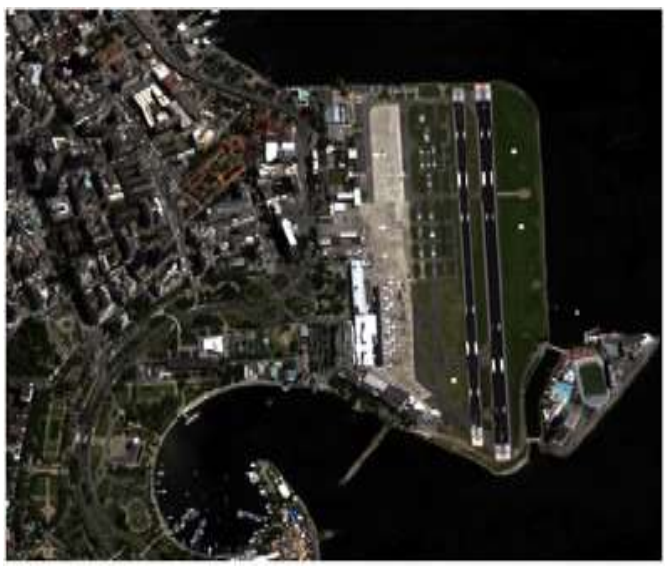

Fig. 3: Selected area, image (II).

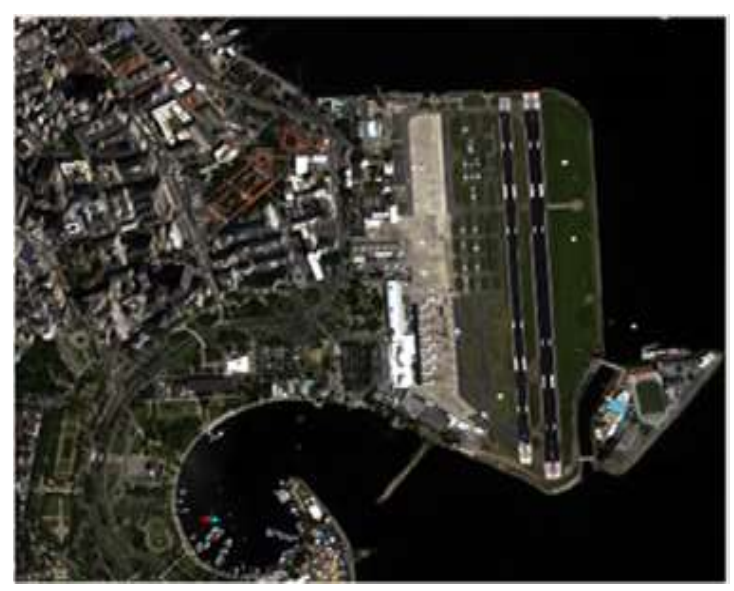

Fig. 4: Selected area, image (III) 


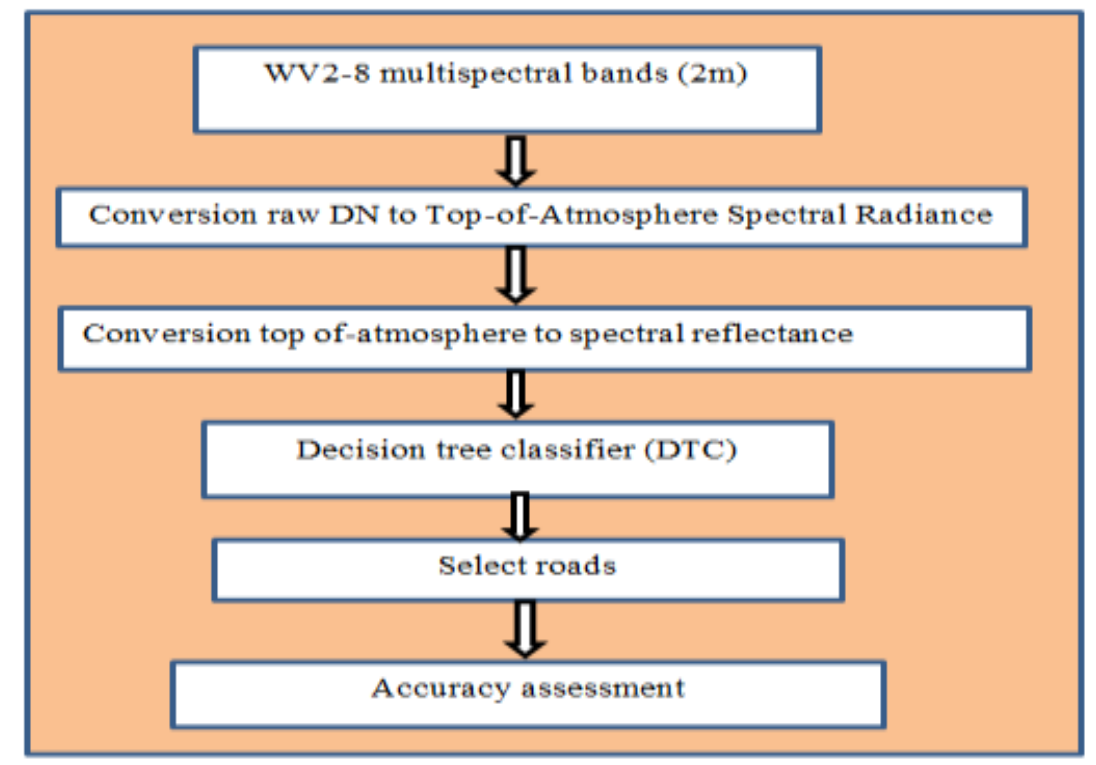

Fig. 5: Experiment steps.

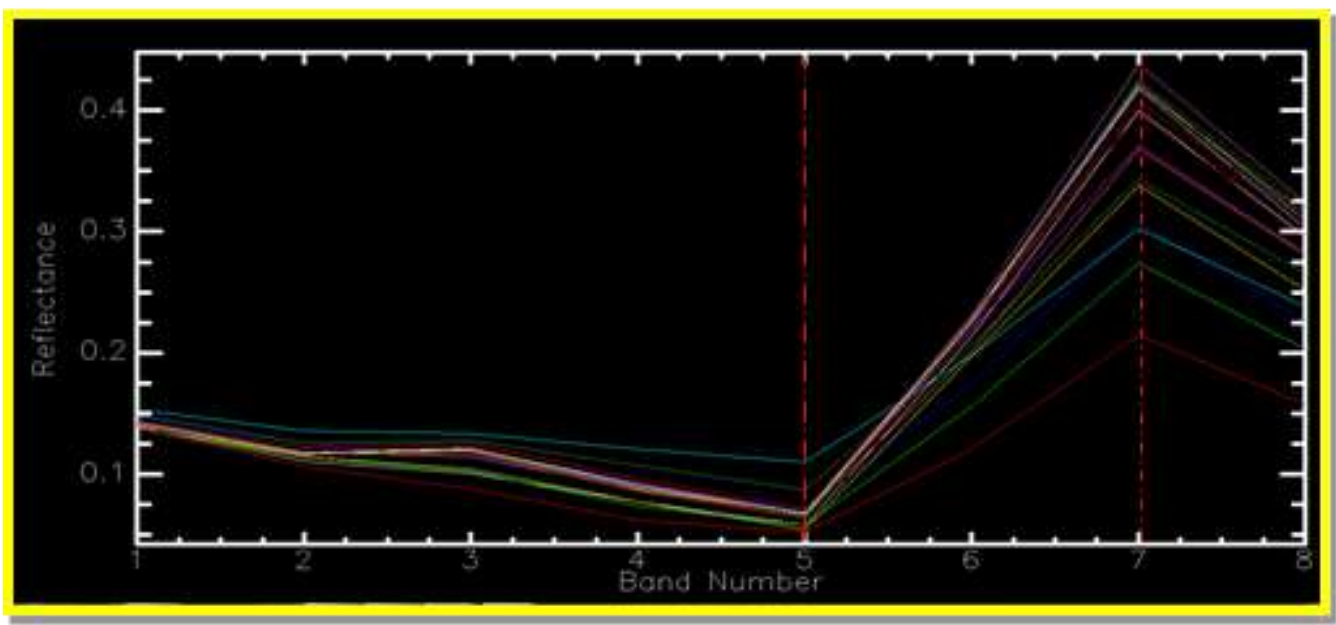

Fig. 6: Spectral reflectance of vegetation class.

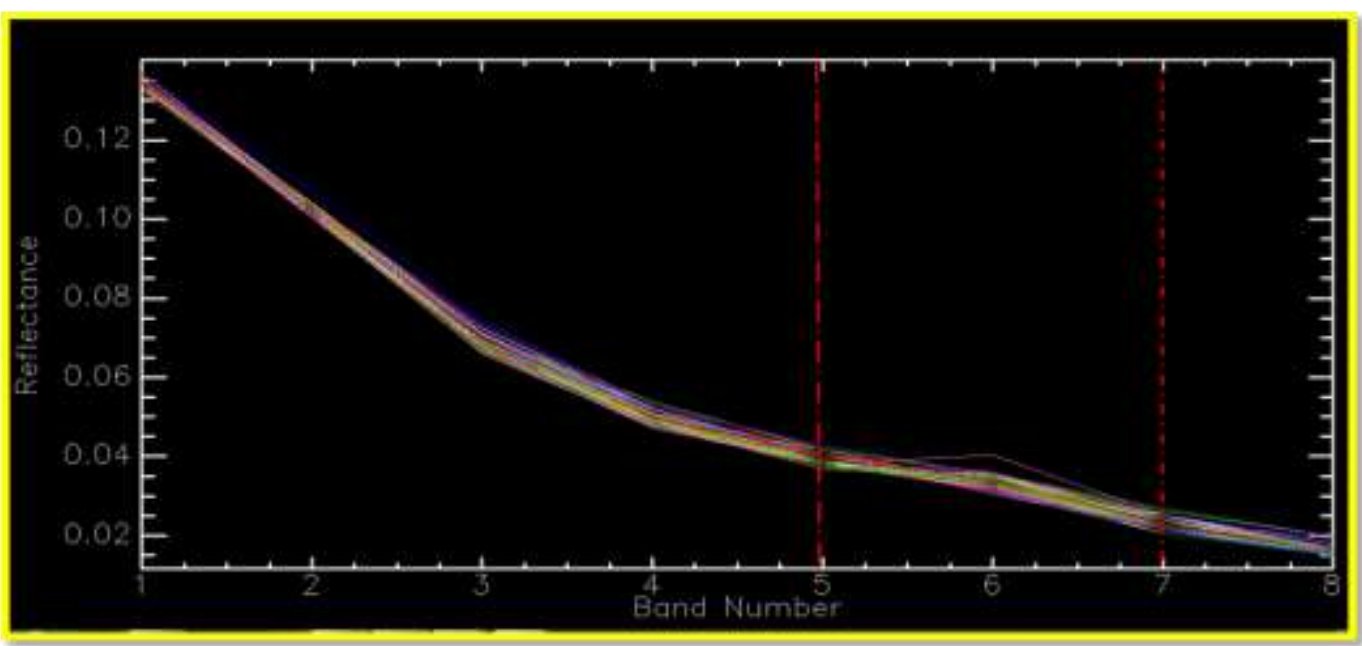

Fig. 7: Spectral reflectance of water class 


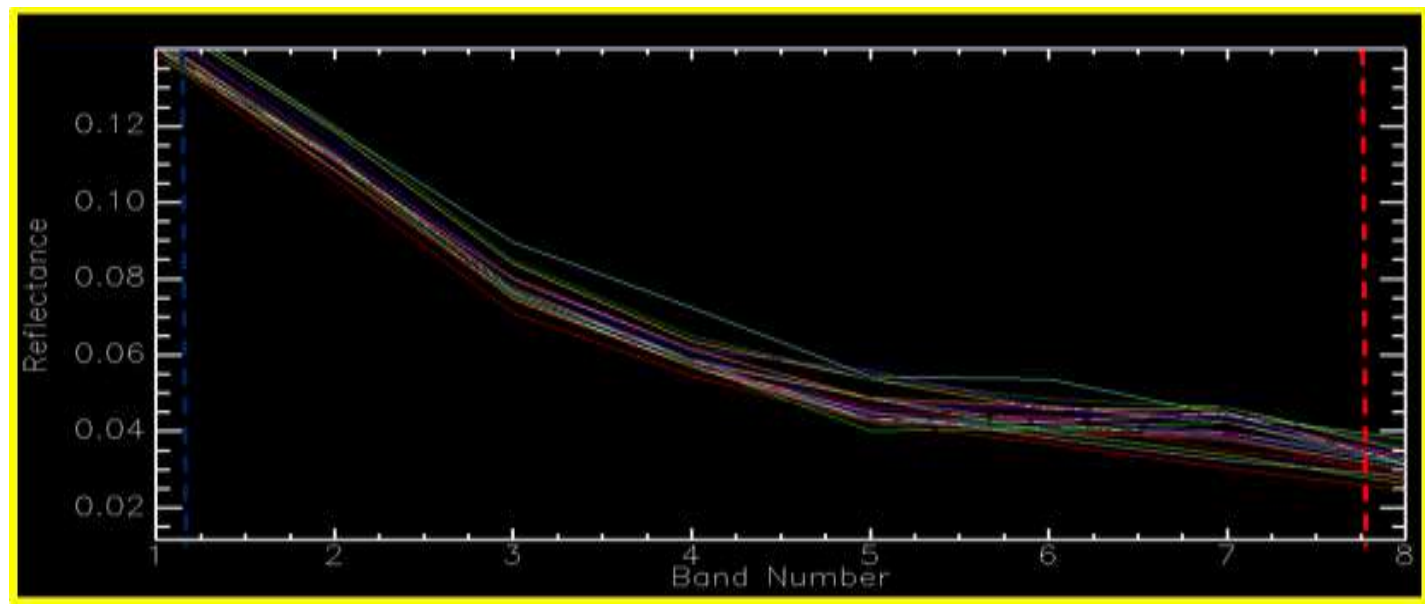

Fig. 8: Spectral reflectance of shadow class

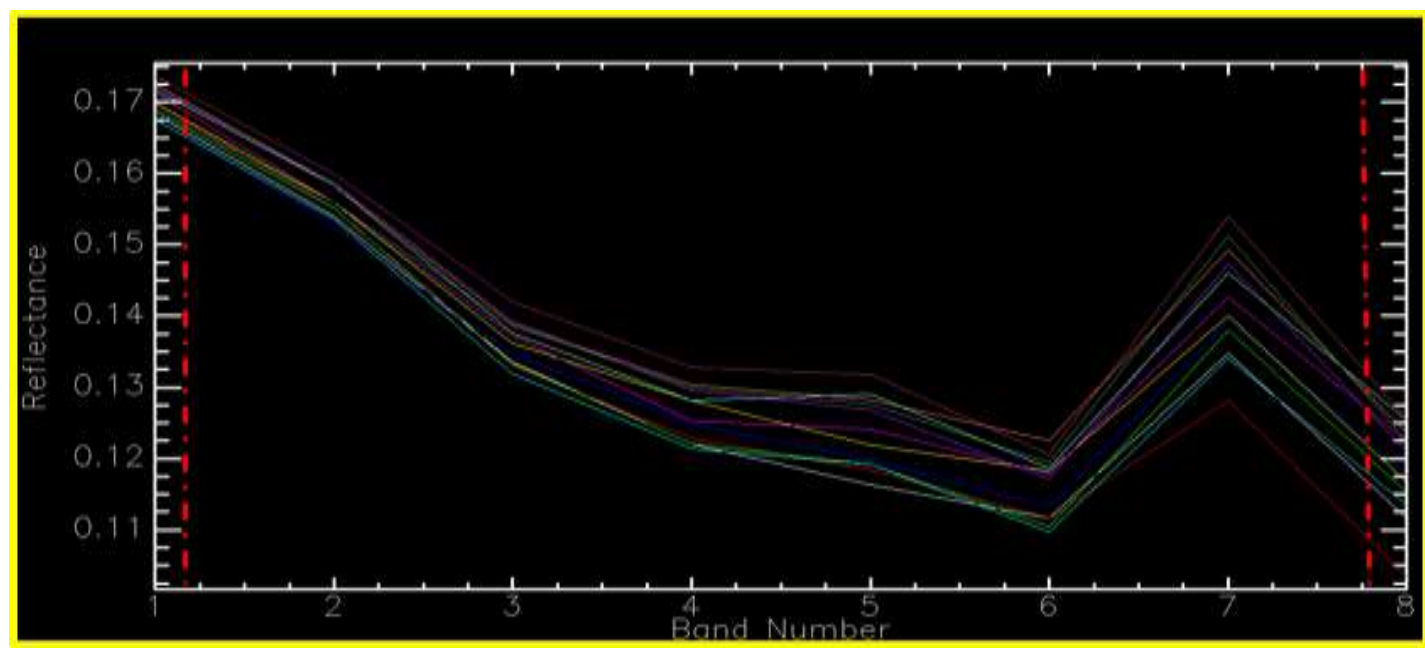

Fig. 9: Spectral reflectance of building class

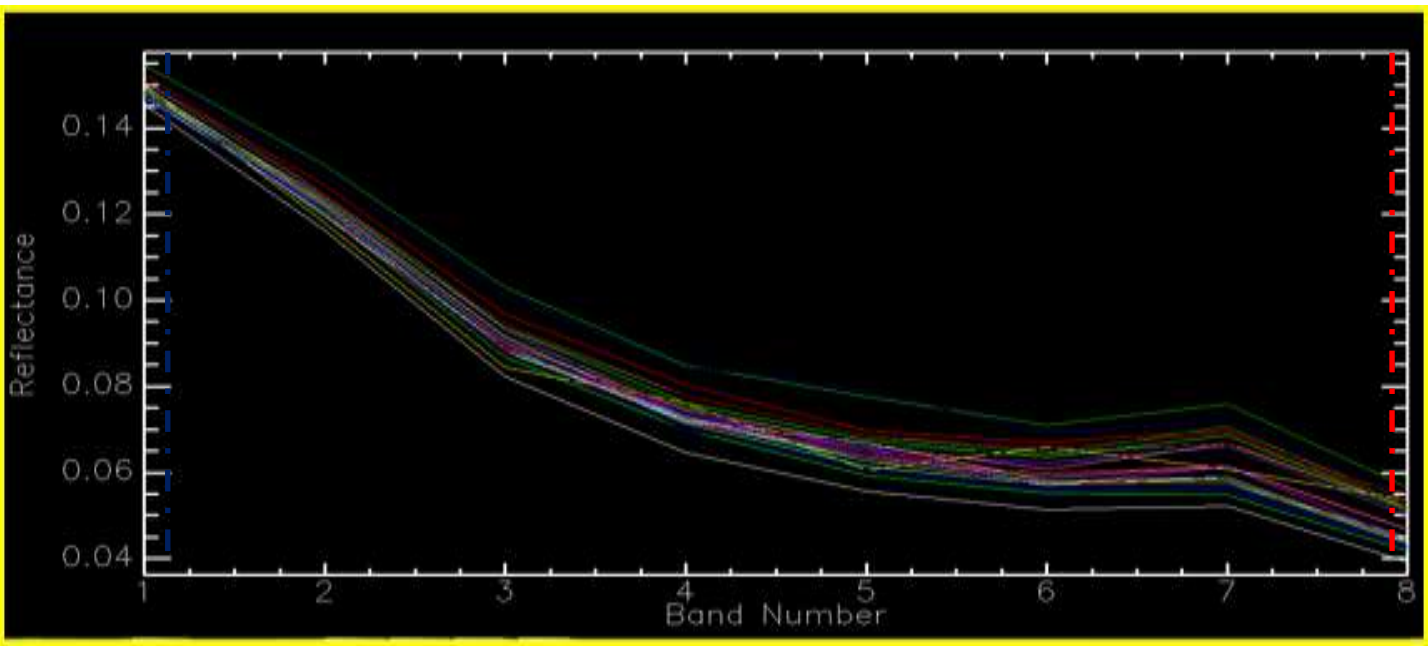

Fig. 10: Spectral reflectance of roads class 


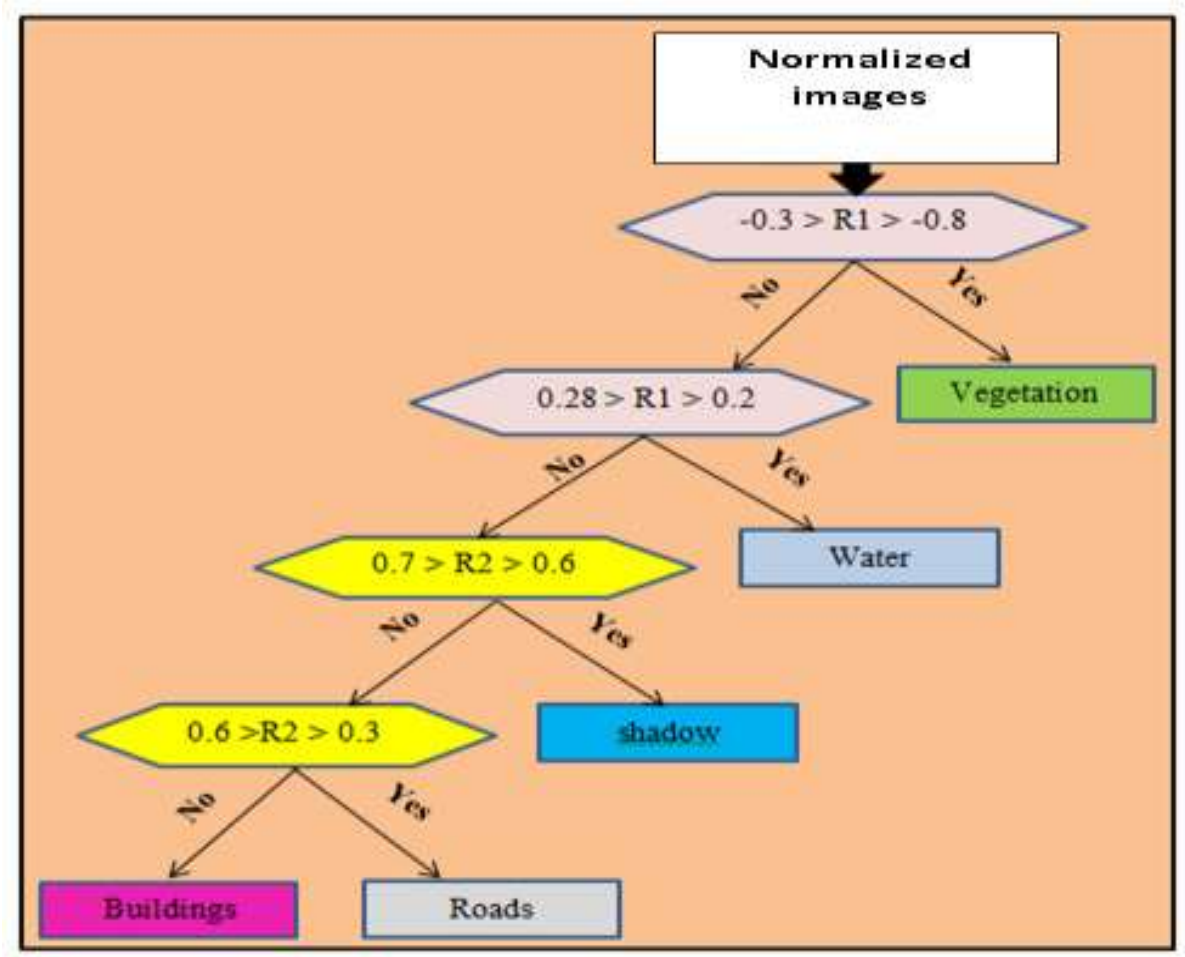

Fig. 11: Feature extraction according to threshold value

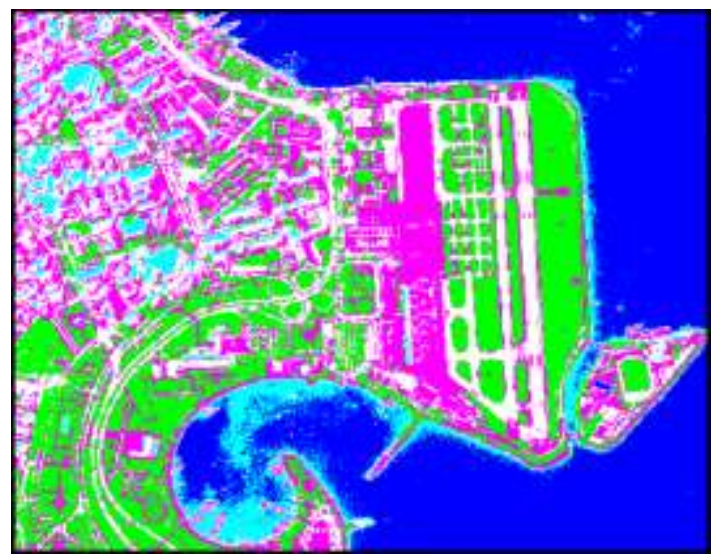

Fig. 12: DTC output for image (I)

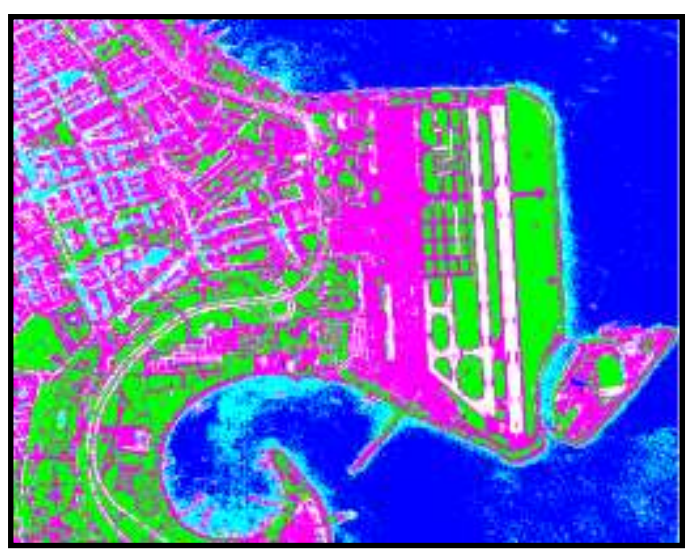

Fig. 13: DTC output for image (II) 


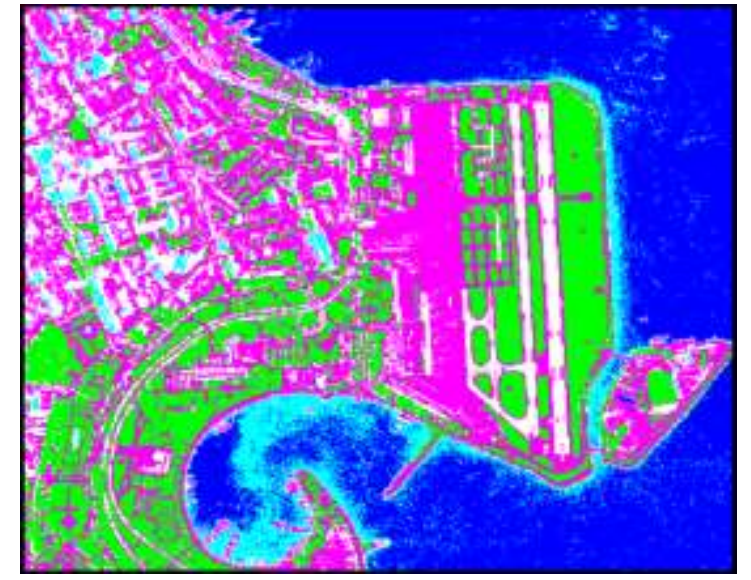

Fig. 14: DTC output for image (III)
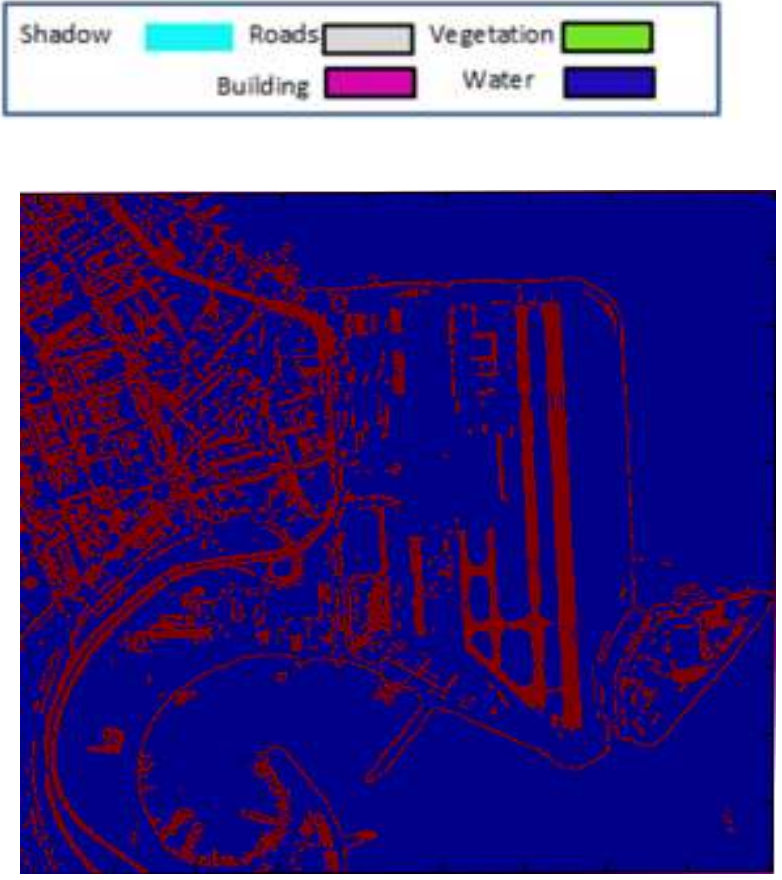

Fig. 15: roads vector layer

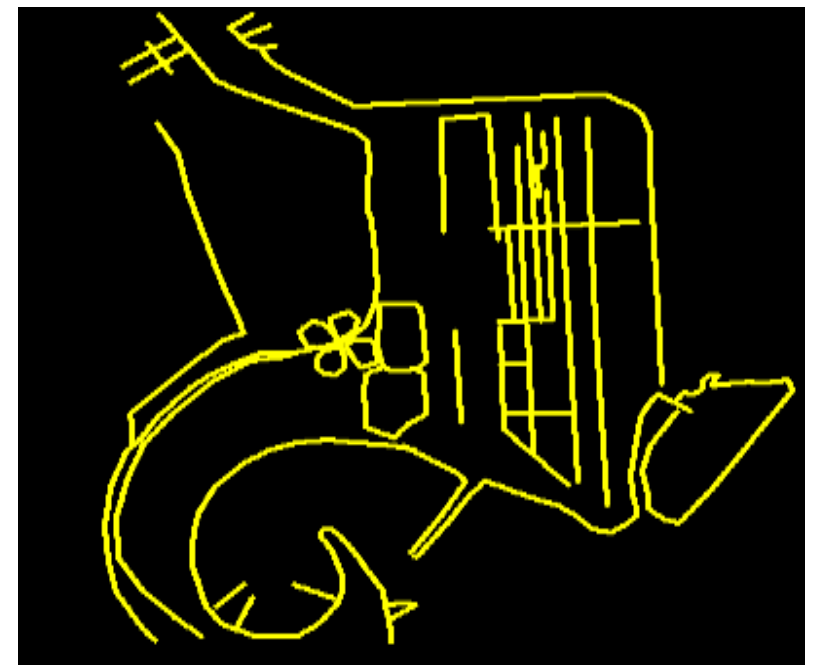

Fig. 16: digitized reference roads 


\section{Reference}

[1] W. Shi, Z. Miao, and J. Debayle, "An integrated method for urban main-road centerline extraction from optical remotely sensed imagery," IEEE Transactions on Geoscience and Remote Sensing, vol. 52, pp. 3359-3372, 2014.

[2] W. Wang, N. Yang, Y. Zhang, F. Wang, T. Cao, and P. Eklund, "A review of road extraction from remote sensing images," Journal of Traffic and Transportation Engineering (English Edition), vol. 3, pp. 271-282, 2016.

[3] C. Vaiphasa, S. Piamduaytham, T. Vaiphasa, and A. K. Skidmore, "A Normalized Difference Vegetation index (NDVI) Time-series of idle agriculture lands: A preliminary study," Engineering Journal, vol. 15, 2011.

[4] X. Zheng, X. Sun, K. Fu, and H. Wang, "Automatic annotation of satellite images via multifeature joint sparse coding with spatial relation constraint," IEEE Geoscience and Remote Sensing Letters, vol. 10, pp. 652-656, 2013.

[5] D. Herumurti, K. Uchimura, G. Koutaki, and T. Uemura, "Urban road extraction based on hough transform and region growing," in Frontiers of Computer Vision,(FCV), 2013 19th Korea-Japan Joint Workshop on, 2013, pp. 220-224.

[6] G. Vosselman and J. d. Knecht, "Road tracing by profile matching and Kalman filtering," Automatic Extraction of Man-Made Objects from Aerial and Space Images, vol. 1, pp. 265-274, 1995.

[7] M. Elhabiby, A. Elsharkawy, and N. El-Sheimy, "Assessment Of Relative Atmospheric Normalization For Multi-Temporal Worldview-2 Data," Assessment, vol. 1, pp. 01-10, 2012.

[8] L. Geosystems, "ERDAS imagine," Atlanta, Georgia, 2004.

[9] A. Elsharkawy, M. Elhabiby, and N. El-Sheimy, "New Combined Object-Based Technique for Efficient Urban Classsification Using WORLDVIEW-2 Data," ISPRS-International Archives of the Photogrammetry, Remote Sensing and Spatial Information Sciences, vol. 1, pp. 191-195, 2012.

[10]T. Updike and C. Comp, "Radiometric use of WorldView-2 imagery," Technical Note, pp. 1-17, 2010.

[11]C. Heipke, H. Mayer, C. Wiedemann, and O. Jamet, "Evaluation of automatic road extraction," International Archives of Photogrammetry and Remote Sensing, vol. 32, pp. 151-160, 1997.

[12]W. Song, T. L. Haithcoat, and J. D. Hipple, "An integrated approach of automatic road extraction and evaluation from remotely sensed imagery," University of Missouri-Columbia, 2001. 